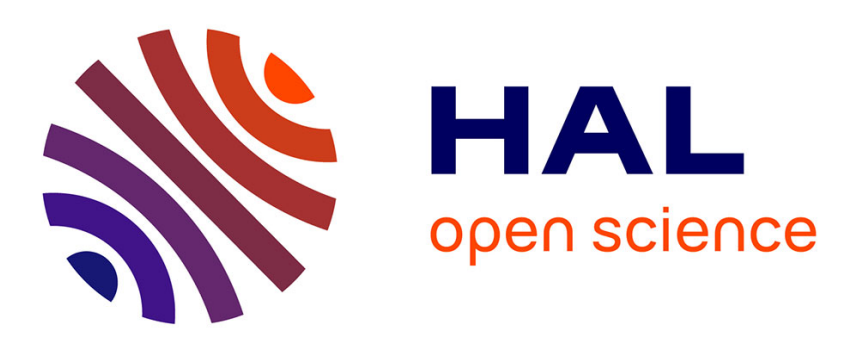

\title{
L'immunothérapie antirabique passive d'hier et d'aujourd'hui.
}

Hervé Bourhy, Laurent Dacheux, Florence Ribadeau-Dumas

\section{To cite this version:}

Hervé Bourhy, Laurent Dacheux, Florence Ribadeau-Dumas. L'immunothérapie antirabique passive d'hier et d'aujourd'hui.. Biologie Aujourd'hui, 2010, 204 (1), pp.71-80. 10.1051/jbio/2009049 . pasteur-01491945

HAL Id: pasteur-01491945

https://hal-pasteur.archives-ouvertes.fr/pasteur-01491945

Submitted on 17 Mar 2017

HAL is a multi-disciplinary open access archive for the deposit and dissemination of scientific research documents, whether they are published or not. The documents may come from teaching and research institutions in France or abroad, or from public or private research centers.
L'archive ouverte pluridisciplinaire HAL, est destinée au dépôt et à la diffusion de documents scientifiques de niveau recherche, publiés ou non, émanant des établissements d'enseignement et de recherche français ou étrangers, des laboratoires publics ou privés. 
Biologie Aujourd'hui, 204 (1), 71-80 (2010)

(C) Société de Biologie, 2010

DOI: $10.1051 /$ jbio/2009049

\title{
L'immunothérapie antirabique passive d'hier et d'aujourd'hui
}

\author{
Hervé Bourhy, Laurent Dacheux et Florence Ribadeau-Dumas \\ Centre National de Référence de la Rage, Centre Collaborateur de l'Organisation Mondiale de la Santé de Référence \\ et de Recherche pour la Rage, Unité Dynamique des Lyssavirus et Adaptation à l'Hôte, Institut Pasteur, \\ 25-28 rue du Docteur Roux, 75724 Paris Cedex 15, France
}

Auteur correspondant : Hervé Bourhy, herve.bourhy@pasteur.fr

Reçu le 27 juin 2009

Résumé - La rage est une maladie mortelle transmise par un animal enragé par morsure, griffure ou léchage sur muqueuse ou peau lésée. La prévention de l'infection après exposition ou prophylaxie post-exposition (PPE) du sujet sans antécédent de vaccination antirabique est basée sur les recommandations de l'OMS et repose sur le nettoyage de la plaie, sur la vaccination du patient et sur une immunothérapie passive basée sur des immunoglobulines antirabiques (RIG) ou leurs dérivés en cas d'exposition à un risque de catégorie 3. La plupart des produits actuels, en particulier les immunoglobulines d'origine humaine et les fragments $F\left(a b^{\prime}\right)_{2}$ d'origine équine, présentent une très bonne efficacité et un taux de réactions adverses minime. Bien que l'intérêt de l'immunothérapie antirabique passive ait été démontré dès la première moitié du $20^{e}$ siècle, le manque d'accessibilité à ces produits pour les populations à risques persiste de nos jours. Il est donc urgent d'informer le personnel et les autorités de santé sur l'intérêt d'utiliser l'immunothérapie passive pour le succès de la PPE et sur l'absence de risque lié à l'usage des produits associés. Dans l'avenir, il faut espérer une augmentation des quantités disponibles de produits purifiés d'origine équine et parallèlement une baisse de leur coût ainsi que la mise sur le marché de nouveaux produits issus des anticorps monoclonaux et du génie génétique dont le coût pourrait être moindre.

Mots clés : Rage / immunothérapie antirabique passive / réactions anaphylactiques / anticorps monoclonaux

\begin{abstract}
The use of passive rabies immunotherapy: from the past to the future.
Rabies is a fatal disease transmitted by infected animals by bite, scratch, licking on broken skin or contamination of mucosis by saliva. The regimen of post-exposure prophylaxis for people not previously vaccinated, that is currently recommended by WHO, consists of a combination of wound cleaning, active immunization and passive immunization when the exposure is of category 3. Most of the products available on the market, in particular human rabies immunoglobulins, highly purified equine rabies immunoglobulins and the derived $F\left(a^{\prime}\right)_{2}$ fragments, are now characterized by high potency and safety. Although the interest of passive anti-rabies immunization was first demonstrated in the first half of the $20 \mathrm{th}$ century, there is still an inadequate supply of these products to the target populations mostly in developing countries. Therefore, it is urgent to set-up training and information actions for healthcare personnel on the need to use passive immunotherapy and the lack of adverse effects of the related products. For the future, we hope that a scale up of production and a lower price will improve the accessibility to these products. The development of new products based on monoclonal antibodies and molecular biology, and which may be cheaper, is promising.
\end{abstract}

Key words: Rabies / passive immunotherapy / anaphylactic reactions / monoclonal antibodies 


\section{Introduction}

La rage est une zoonose virale à laquelle sont sensibles tous les mammifères. Elle est due à un virus de la famille des Rhabdoviridae et du genre Lyssavirus qui présente un tropisme important pour les cellules nerveuses. Elle est transmissible accidentellement à l'Homme, généralement à la suite d'une morsure par un animal enragé.

Plus de 120 ans après la découverte du principe de la prophylaxie post-exposition (PPE) par Louis Pasteur, le contrôle de la rage reste une des priorités de l'Organisation Mondiale de la Santé (OMS). En effet, si la rage semble régresser en Europe du fait de la vaccination orale des renards, il ne faut pas sous-estimer la gravité de cette infection dans certaines parties du monde moins favorisées. Le nombre de décès humains dans le monde dus à la rage est estimé à environ 50000 par an (Knobel et al., 2005). Parallèlement, environ 15 millions de PPE sont administrées chaque année (Bourhy et al., 2009). En France, la rage vulpine apparue en 1968 dans l'est a été éradiquée en 1998. Les problèmes actuels posés par la rage sont liés à l'identification d'un cycle épidémiologique particulier chez les chiroptères, mais surtout à l'importation illégale d'animaux de compagnie ou sauvages en phase d'incubation, et au risque d'infection chez les voyageurs dans les pays d'enzootie rabique (Bourhy et al., 2005).

La rage se caractérise par l'apparition d'un tableau clinique d'encéphalite dont les symptômes sont très variables selon les individus et les espèces considérés mais dont l'issue est toujours une mort rapide hormis de rares exceptions. Le diagnostic précoce de l'infection n'est pas possible chez l'Homme (Dacheux et al., 2008). La prévention de la maladie repose donc sur la surveillance de l'animal mordeur lorsqu'elle est possible et sur l'administration d'une PPE. Celle-ci est toujours efficace lorsqu'elle suit les recommandations de l'OMS, à de rares exceptions près (Anonymous, 2005; WHO, 2007; Wilde, 2007). Il n'y a pas de traitement curatif de la rage déclarée et le diagnostic différentiel avec d'autres encéphalites virales est souvent difficile. Dans ce contexte la prévention préexposition et la PPE revêtent donc une importance particulière. Parallèlement, les recherches sur la prise en charge efficace de la rage déclarée se poursuivent (Willoughby et al., 2005; Coutard et al., 2008).

La PPE associe le vaccin, fabriqué sur cultures cellulaires et bien toléré, et selon les cas, une immunothérapie passive constituée d'immunoglobulines spécifiques ou de leurs dérivés, d'origine humaine ou équine. L'usage de ces produits est réservé exclusivement à l'immunisation post-exposition des sujets sans antécédents de vaccination antirabique. Ils sont utilisés pour obtenir rapidement des concentrations d'anticorps neutralisants efficaces, en attendant la mise en place de la réponse immunitaire active qui suit la vaccination.

\section{Historique}

La première mention de l'intérêt des immunoglobulines antirabiques (RIG) dans le traitement de la rage apparaît en 1889. Babes et Lepp rapportent qu'une protection passive contre la rage peut être transférée à des chiens ou à des lapins avec du sang de chiens hyperimmunisés contre la rage (1889). La notion de séro-neutralisation du virus rabique in vitro par du sang de chien hyperimmunisé est introduite par Babes et Cherchez en 1891. Ces travaux sont par la suite confirmés dans des modèles expérimentaux de rage utilisant la souris, le cobaye et le singe traités avec un sérum hyperimmun obtenu chez le lapin par Habel (1945) puis par Koprowski et ses collaborateurs (1950).

Proca et Bobès soulignent dès 1940 l'utilité chez l'Homme de l'immunothérapie passive en association avec le vaccin dans les morsures de la face. L'importance de cette sérothérapie chez l'homme est finalement démontrée en Iran (Baltazard \& Bahmanyar, 1955; Bahmanyar et al., 1976; 2000; Bahmanyar, 1978) et en URSS (Selimov et al., 1959). Par la suite une série d'études coordonnées par l'OMS ont permis de déterminer les modalités pratiques de l'application des RIG. Elles ont fixé en particulier les doses (toujours en vigueur) à injecter (Atanasiu et al., 1956, 1957, 1961, 1967). Le premier (en 1950) (WHO, 1950), (figure 1) puis le deuxième (en 1954) rapports du Comité d'experts de la rage de l'OMS (OMS, 1954), recommandent de procéder à une injection de sérum hyperimmun après exposition à l'infection. Malheureusement, à cette époque, de nombreuses réactions anaphylactiques sont enregistrées (Karliner \& Belaval, 1965) laissant souvent le médecin devant un dilemme : utiliser les RIG et encourir les risques de chocs anaphylactiques ou ne pas les utiliser et risquer un échec de traitement. Pour limiter ce risque, il était conseillé d'utiliser la méthode de Besredka et d'administrer des antihistaminiques.

Il existait pourtant une solution simple qui mit du temps à s'imposer. Dès 1908, Marie discutait l'intérêt de l'usage d'un sérum antirabique homologue (Marie, 1908). Plusieurs modes de préparation furent utilisés mais le titre des sérums obtenus restait faible (Hosty et al., 1959; Anderson \& Sgouris, 1965; Sikes, 1969; Winkler et al., 1969). L'interférence entre le vaccin et le sérum antirabique posait des problèmes (Archer \& Dierks, 1968; Wiktor et al., 1971). Sikes étudia chez le chien l'interférence liée à l'injection à distance mais simultanée du sérum hyperimmun et du vaccin (Sikes, 1969). Il en conclut que la demi-vie de l'anticorps 
«Depuis de nombreuses années, on reconnait la possibilité de l'emploi prophylactique du sérum antirabique, et de nombreuses expériences en ont fait entrevoir les possibilités pratiques. Des expériences plus récentes, fondées sur des méthodes quantitatives, ont montré nettement la supériorité du sérum hyperimmun - surtout lorsqu'il est associé à la vaccinothérapie - sur l'emploi de la seule vaccination, en cas d'infection par le virus des rues par voie périphérique. On peut maintenant produire ce sérum en employant de gros animaux tels que le mouton, et concentrer l'anticorps spécifique. L'emploi d'un sérum hyperimmun extrêmement actif, avant traitement par le vaccin, fait naître l'espoir de sauver la plupart des hommes contaminés par le virus rabique et chez lesquels la période d'incubation serait trop brève pour que puisse se développer une immunité active. Ces observations expérimentales conduisent le comité à penser qu'actuellement le sérum associé au vaccin serait le moyen le plus efficace d'empêcher l'éclosion de la rage après contamination grave, et à recommander très vivement que soit instituée une expérience sur le terrain portant sur l'homme exposé à l'infection dans de telles conditions. »

Fig. 1. Recommandations du premier Comité d'Experts de la Rage de l'OMS en 1950 (WHO, 1950).

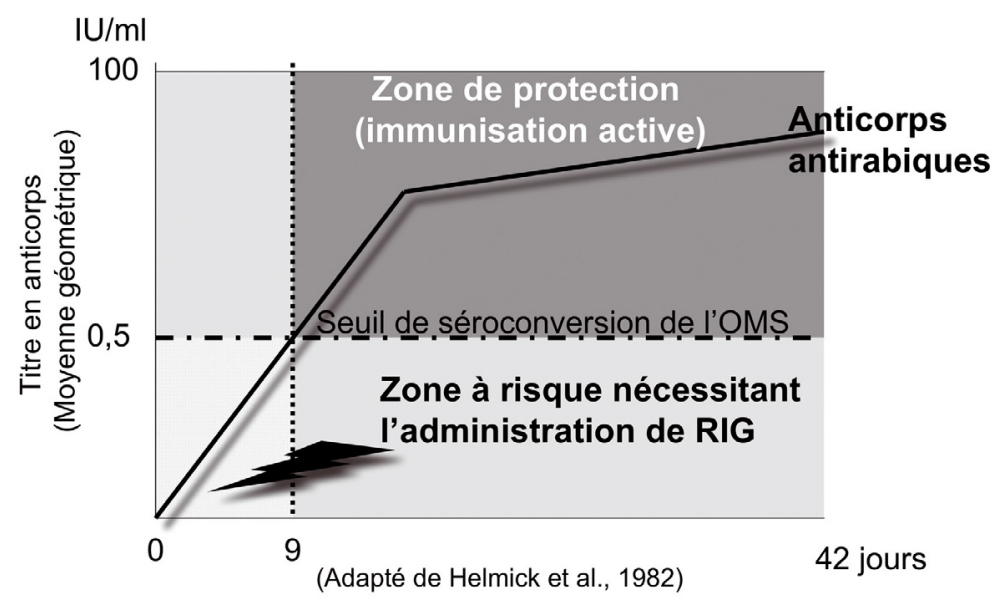

Fig. 2. Représentation schématique de l'action de l'immunothérapie antirabique passive.

hétérologue était d'un jour et demi, et celle de l'anticorps homologue de sept jours. C'est Cabasso qui, dans les années 1970, standardisa la production et fixa les doses d'anticorps homologues à utiliser (Cabasso, 1974; Cabasso et al., 1971, 1974). Ces doses furent par la suite confirmées (Hattwick et al., 1974) et ces recommandations furent alors adoptées par le sixième Comité d'experts de la rage de l'OMS (WHO, 1973).

Rapidement, l'intérêt de l'utilisation des RIG en infiltration locale au niveau de la morsure fut démontré (Kaplan et al., 1962; Dean et al., 1963; Kaplan \& Paccaud, 1963; Dean, 1966). La rétention des RIG d'origine humaine radio-marquées au site d'injection pendant au moins 24 heures fut démontrée en 2000 (Saesow et al., 2000). La recommandation d'utiliser la dose de RIG au maximum en infiltration locale au niveau du site de morsure fut entérinée lors du septième Comité d'experts de la rage de l'OMS (Anonymous, 2005).

Parallèlement, des progrès concernant la qualité des sérums xénogéniques furent accomplis. Traditionnellement, les sérums équins sont concentrés et purifiés par précipitation au sulfate d'ammonium ou par fractionnement à l'aide d'acide caprylique. La purification peut aussi s'effectuer par chromatographie par échange d'ions, par chromatographie par interaction hydrophobe, ou par chromatographie par protéine A ou protéine $\mathrm{G}$. Cependant ces techniques efficaces restent très coûteuses dans le processus industriel. Aujourd'hui elles sont appliquées avec succès aux fragments $\mathrm{F}(\mathrm{ab})_{2}$ obtenus après traitement du sérum à la pepsine (Fernandes et al., 2008; Quiambao et al., 2008).

\section{Les immunoglobulines antirabiques utilisées aujourd'hui}

Deux produits confèrent une immunité passive immédiate permettant d'attendre la montée de la réponse immune active liée à la vaccination dans les expositions de catégorie 3 (figures 2, 3 et 4) :

- les RIG d'origine équine (ERIG) : extraites et purifiées du sérum de chevaux hyperimmunisés, elles sont efficaces et bon marché. Elles sont bien supportées (Lang et al., 1998). Le test de sensibilité cutanée n'est pas prédictif et n'est plus recommandé (Cupo et al., 2001). Des fragments F(ab') dérivés de ces produits sont maintenant sur le marché (Quiambao et al., 2008) ; 
A

Protocoles validés par l'OMS: nombre de doses de vaccins par voie IM et immunothérapie passive (RIG)

B

ERIG: $40 \mathrm{UI} / \mathrm{kg}$

\begin{tabular}{|l|l|l|l|l|l|}
\hline D0 & $\mathrm{D}_{3}$ & $\mathrm{D}_{7}$ & $\mathrm{D} 14$ & $\mathrm{D} 21$ & $\mathrm{D} 28$ \\
\hline
\end{tabular}

ESSEN:

$1 \mathrm{x} \quad 1 \mathrm{x} \quad 1 \mathrm{x} \quad 1 \mathrm{x} \quad 1 \mathrm{x}$

ZAGREB:

$1 \mathrm{x} \quad 1 \mathrm{x}$

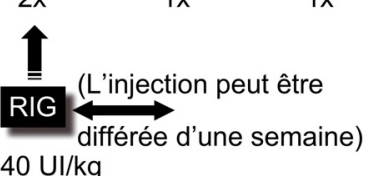

HRIG: $20 \mathrm{UI} / \mathrm{kg}$

(En infiltration locale, le reste en IM à distance)

Que choisir?
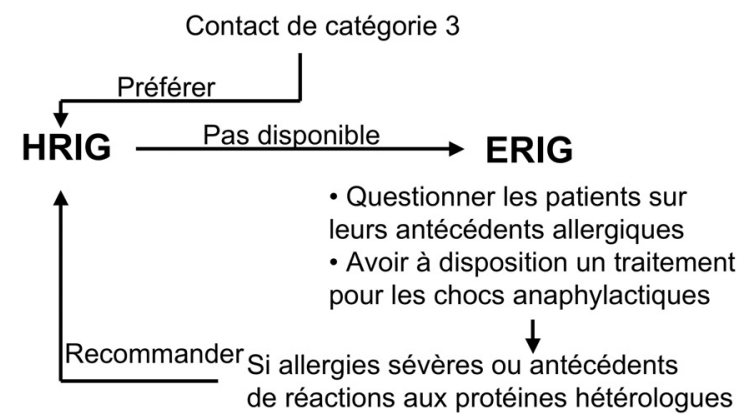

Fig. 3. Différents produits disponibles et leur utilisation.

- les RIG d'origine humaine (HRIG) : elles sont préparées à partir du plasma obtenu de sujets vaccinés contre la rage et donneurs volontaires. Elles sont très efficaces, parfaitement supportées mais elles sont rares et chères (Helmick et al., 1982). Le procédé de fabrication doit garantir l'absence de contaminant (virus HBV, HCV, HIV, etc.).

Il faut noter que ces produits, même injectés très précocement, ne peuvent à eux seuls éliminer les risques d'infection. Ils doivent donc impérativement être associés à la vaccination. Dans ce cas et si les recommandations de l'OMS sont suivies, la protection conférée est quasi totale.

\section{Modes de production}

\section{- ERIG}

Les ERIG sont produites par immunisation de chevaux ou de mules par voie sous-cutanée pendant une période d'environ deux mois avec une souche vaccinale de virus rabique inactivée par la $\beta$-propiolactone. Parfois les animaux subissent des injections par voie sous-cutanée utilisant des doses croissantes de virus vivants. Les titres en anticorps sont vérifiés périodiquement. Seuls les animaux montrant une réponse satisfaisante en anticorps sont gardés. Des variantes existent, en particulier une réponse plus rapide est obtenue par l'injection concomitante d'adjuvant de Freund, d'hydroxide d'alumine ou de bétonite (Luekrajang et al., 1999). Le sérum antirabique ainsi obtenu est ensuite concentré et purifié. Pour ce faire, une précipitation au sulfate d'ammonium est utilisée. Certaines variantes adjoignent une digestion enzymatique préalable et poursuivent par une thermo-coagulation pour éliminer l'excès de protéines (Glück \& Labert, 1999). Une digestion à la pepsine suivie d'une précipitation au sulfate d'ammonium et d'une purification par chromatographie par échange d'ions permet d'obtenir des fragments $\mathrm{F}(\mathrm{ab})_{2}$ hautement purifiés (Quiambao et al., 2008).

\section{- HRIG}

L'utilisation d'immunoglobulines homologues élimine les risques de choc anaphylactique (immédiat et dramatique) et de maladie sérique (manifestation retardée), associés aux immunoglobulines hétérologues. Les donneurs sont choisis parmi ceux présentant les titres en anticorps antirabiques neutralisants les plus élevés après vaccination préventive ou PPE. Ils reçoivent alors plusieurs doses de rappel avant la première récolte d'immunoglobulines qui se fait par plasmaphérèse.

\section{Le rôle de la glycoprotéine}

Le genre Lyssavirus est subdivisé en sept génotypes. On distingue pour chaque génotype un virus prototype : le virus de la rage (RABV) ou génotype 1 qui comprend toutes les souches vaccinales, le virus $L a$ gos bat (LBV, génotype 2), le virus Mokola (MOKV, génotype 3), le virus Duvenhage (DUVV, génotype 4), les virus EBLV-1 (pour European Bat Lyssavirus Subtype 1) (génotype 5) et EBLV-2 (génotype 6), et le virus ABLV (pour Australian Bat Lyssavirus). Les génotypes 2, 3, 4, 5, 6 et 7 constituent ce qu'on appelle les virus apparentés à la rage (Delmas et al., 2008). De nombreux autres Lyssavirus ont été récemment mis en évidence à partir de chauves-souris en Eurasie (Kuzmin et al., 2008). Ils ne sont pas encore classés.

La morphologie des Lyssavirus est caractéristique et correspond à celle d'un obus ou d'une balle de revolver, mesurant environ $60 \mathrm{~nm}$ de large sur 120 à $180 \mathrm{~nm}$ de long. Ce sont des virus enveloppés, fragiles et sensibles aux solvants des lipides, au savon, etc. (intérêt de la désinfection sans délai des plaies afin d'inactiver le virus), constitués d'un ARN monocaténaire non segmenté de polarité négative d'environ $12 \mathrm{~kb}$ et de cinq protéines virales (la nucléoprotéine $\mathrm{N}$, la phosphoprotéine $\mathrm{P}$, la protéine de matrice $\mathrm{M}$, la 


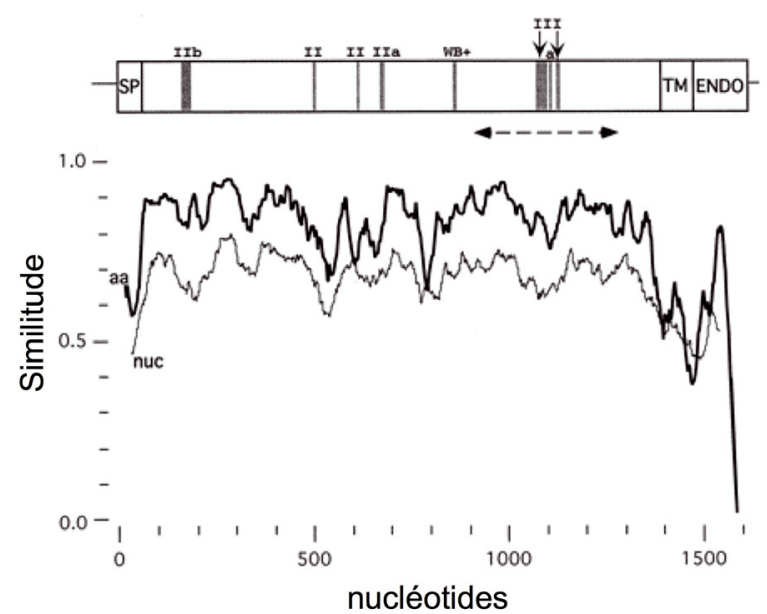

SP, peptide signal ;

TM, région transmembranaire;

ENDO, région intracytoplasmique.

Fig. 4. Représentation schématique des sites antigéniques de la glycoprotéine du virus rabique et de leur variabilité (d'après Badrane et al., 2001).

glycoprotéine $\mathrm{G}$ et la polymérase $\mathrm{L}$ ). La protéine $\mathrm{G}$ est retrouvée sous forme de spicules trimériques insérés à la surface de l'enveloppe virale. Elle induit la synthèse d'anticorps neutralisants protecteurs. L'intégrité du virion est essentielle à l'obtention d'un titre en anticorps élevé.

Sept sites antigéniques indépendants impliqués dans la réponse lymphocytaire $B$ ont été identifiés sur la protéine $\mathrm{G}$ (figure 4). La détermination précise des sites dans le système immunitaire murin a été obtenue par l'étude de la réactivité des anticorps monoclonaux $(\mathrm{AcM})$ vis-à-vis de la glycoprotéine entière ou de ses fragments peptidiques et par la caractérisation de mutants d'échappement vis-à-vis de ces AcM. Les sites II (AA 34-42 et 198-200) et III (AA 330-338 et AA 357) sont qualifiés de sites antigéniques majeurs car ils concentrent la majorité de la réactivité antigénique. Les sites I (AA 231), IV (AA 264) et V sont des épitopes indépendants. Le site a (AA 342-343) est un site mineur (Benmansour et al., 1991). Le site VI (AA 264) est un site non conformationnel et reconnu par le système immunitaire de l'homme (Dietzschold et al., 1990; Lafon et al., 1990). Des peptides synthétiques ont aussi servi à la délimitation in vitro des sites impliqués dans les fonctions $\mathrm{T}$ auxiliaire et $\mathrm{T}$ cytotoxique des lymphocytes. Un site (R21, AA 18-44) impliqué dans la réponse $\mathrm{T}$ auxiliaire a ainsi été défini. La glycoprotéine virale, et en particulier ses sites antigéniques, sont très variables d'un génotype à l'autre. En conséquence, les anticorps neutralisants obtenus par immunisation avec une souche vaccinale de génotype 1 ont une activité réduite quand on s'éloigne de ce génotype (Hanlon et al., 2005).

En conclusion, la glycoprotéine est la cible de la majeure partie de la production d'anticorps neutralisants. Néanmoins, sa variabilité antigénique limite l'efficacité de ces anticorps en cas d'exposition à des isolats très éloignés antigéniquement de ceux contre lesquels ils sont ciblés. Ce risque est néanmoins minime car plus de $98 \%$ des cas humains dans le monde sont dus à des virus canins de génotype 1 .

\section{Les produits actuels et les recommandations d'usage de l'OMS}

La PPE doit être entreprise rapidement au sein de centres de traitement antirabique ou de centres de soin disposant d'un personnel formé. Les premiers gestes à effectuer sans délai après contact sont le lavage abondant à l'eau savonneuse de la blessure pendant plusieurs minutes, l'antisepsie (avec un dérivé iodé par exemple), la désinfection, le parage sans suture (sauf impératif fonctionnel ou esthétique), associés à une prophylaxie antitétanique et une antibiothérapie.

L'immunothérapie passive n'est appliquée que dans les expositions à risque de catégorie 3 (Tableau 1). Les doses recommandées ont été calculées pour ne pas interférer avec l'immunisation active découlant de l'administration simultanée du vaccin (Archer \& Dierks, 1968; Wiktor et al., 1971; Cabasso, 1974; Hattwick et al., 1974). Pour les mêmes raisons, les RIG ou produits dérivés ne doivent pas être administrés avec la même seringue que le vaccin.

L'injection se fait par infiltration soigneuse de la plus grande partie possible de la dose dans la profondeur et autour de la plaie. Le reste de la dose est injectée par voie intramusculaire dans la zone la plus proche possible de la zone contaminée mais différente du site du vaccin antirabique (en raison du risque d'interaction) et différente de la fesse (zone non efficace). Si la taille de la plaie le justifie, le produit est dilué dans du sérum physiologique afin de couvrir 
Tableau 1. Recommandations actuelles du Comité d'experts de la rage de l'OMS concernant les catégories d'exposition à la rage et l'utilisation de l'immunothérapie passive.

\begin{tabular}{|c|c|c|}
\hline Catégorie & $\begin{array}{l}\text { Nature du contact avec } \\
\text { l'animal suspect }^{\text {a }}\end{array}$ & Traitement recommandé \\
\hline \multirow[t]{2}{*}{$\mathbf{I}$} & $\begin{array}{l}\text { Contact direct ou alimentation de } \\
\text { l'animal }\end{array}$ & $\begin{array}{l}\text { Aucun si une anamnèse fiable } \\
\text { peut être obtenue (l'interrogatoire }\end{array}$ \\
\hline & Léchage sur peau intacte & $\begin{array}{l}\text { d'un entant }<6 \text { ans n'est pas } \\
\text { considéré comme fiable) }\end{array}$ \\
\hline II & $\begin{array}{l}\text { Peau découverte mordillée } \\
\text { Griffure bénigne ou excoriation } \\
\text { sans saignement } \\
\text { Léchage sur peau lésée }\end{array}$ & $\begin{array}{l}\text { Vacciner b immédiatement (ou } \\
\text { au moins le plus tôt possible) } \\
\text { Ne pas poursuivre la } \\
\text { vaccination si l'animal est } \\
\text { confirmé négatif contre la rage à } \\
\text { l'issue de la période } \\
\text { d'observation ou si la recherche } \\
\text { de rage au laboratoire par une } \\
\text { technique suffisamment sensible } \\
\text { est négative. }\end{array}$ \\
\hline III $^{\mathbf{c}}$ & $\begin{array}{l}\text { Morsure ou griffure ayant } \\
\text { traversé la peau } \\
\text { Contamination des muqueuses } \\
\text { par la salive (léchage) }\end{array}$ & $\begin{array}{l}\text { Vacciner et appliquer } \\
\text { immédiatement } \\
\text { l'immunothérapie passive. } \\
\text { Ne pas poursuivre la } \\
\text { vaccination si l'animal est } \\
\text { confirmé négatif contre la rage à } \\
\text { l'issue de la période } \\
\text { d'observation ou si la recherche } \\
\text { de rage au laboratoire par une } \\
\text { technique suffisamment sensible } \\
\text { est négative. }\end{array}$ \\
\hline
\end{tabular}

\footnotetext{
${ }^{a}$ L'exposition aux rongeurs, lapins et lièvres ne requiert jamais ou rarement de prophylaxie post-exposition.

b S'il s'agit d'un chien ou d'un chat apparemment en bonne santé vivant dans une zone à risque faible et placé sous observation vétérinaire, le traitement peut être différé.

${ }^{\mathrm{c}} \mathrm{Y}$ compris le cas particulier du contact avec une chauve-souris.
}

l'ensemble de la surface. L'administration du vaccin est commencée simultanément.

Seuls deux types de produits ont une autorisation de mise sur le marché (AMM) en France :

\section{HRIG :}

Imogam Rage ${ }^{\circledR}$ (Sanofi Pasteur). Elle se présente en flacons de 2 ou $10 \mathrm{ml}$ et est dosée au minimum à $150 \mathrm{UI} / \mathrm{ml}$. La posologie recommandée est de $20 \mathrm{UI} / \mathrm{kg}$ de poids corporel. Cette posologie est la même pour toutes les immunoglobulines d'origine humaine.

\section{$>$ Produit issu d'ERIG :}

Favirab $^{\circledR} \mathrm{F}(\mathrm{ab})_{2}$ (Sanofi Pasteur). La posologie est de $40 \mathrm{UI} / \mathrm{kg}$ (de même que pour toutes les immunoglobulines d'origine équine), ce qui correspond à $1 \mathrm{ml}$ de Favirab ${ }^{\circledR}$ pour $5 \mathrm{~kg}$ de poids.

Ces produits doivent être gardés au réfrigérateur entre 2 et $8{ }^{\circ} \mathrm{C}$ (ne pas utiliser une dose qui a été congelée). Une fois injectées, les RIG ont une demi-vie courte, de quelques jours. Enfin elles sont moins, voire peu, efficaces vis-à-vis des génotypes de Lyssavirus autres que le génotype 1 .

\section{Tolérance, contre-indication}

La posologie et le schéma séro-vaccinal sont les mêmes quel que soit l'âge (y compris chez l'enfant). Les immunoglobulines humaines sont très bien tolérées (Suwansrinon et al., 2005, 2007). La fréquence et la gravité des effets secondaires des produits d'origine équine ont diminué depuis que ceux-ci sont purifiés et séparés des fractions responsables des réactions anaphylactiques. C'est en particulier le cas des fragments $\mathrm{F}(\mathrm{ab})_{2}$. Les effets secondaires décrits avec les immunoglobulines (HRIG, ERIG purifiées, $\mathrm{F}\left(\mathrm{ab}{ }^{\prime}\right)_{2}$ ) sont :

- locaux : douleurs au point d'injection, raideur du membre;

- ou généraux : fièvre, malaise, céphalées, rash, œdème angio-neurotonique, syndrome néphrotique, anaphylaxie;

- en tant que produit d'origine plasmatique humaine, les immunoglobulines sont potentiellement à risque infectieux (même si la sélection des donneurs, le contrôle microbiologique et le traitement par la chaleur ou la purification, réduisent de façon drastique ce risque). 
Compte-tenu de la létalité de la rage, il n'existe pas de contre-indication à la PPE. La grossesse et l'allaitement ne sont ainsi pas une contre-indication à la sérovaccination en post-exposition. En revanche, les HRIG interfèrent avec la réponse aux vaccins vivants (varicelle, rougeole, fièvre jaune... ). Il faut donc éviter d'administrer un vaccin vivant dans les quatre mois qui suivent des RIG. Un contrôle sérologique et parfois une revaccination sont nécessaires chez les patients qui reçoivent un vaccin vivant dans les 14 jours consécutifs à une dose d'immunoglobulines.

\section{Les problèmes d'usage dans le monde}

D'après les doses de vaccins consommées dans le monde, on considère que 15 millions de personnes reçoivent une PPE chaque année. Seuls 2 à $5 \%$ des patients débutant une PPE reçoivent des RIG (Bourhy et al., 2009). Selon les estimations de l'OMS, $60 \%$ des patients présentant une exposition de catégorie 3 ne bénéficient malheureusement pas d'une immunothérapie passive. Cette sous-utilisation est liée à plusieurs raisons. Le coût des produits disponibles, à la charge des patients dans de nombreux pays, est parfois dissuasif. Les quantités produites localement sont souvent insuffisantes. Enfin, la disponibilité de ces produits dans les dispensaires locaux n'est souvent pas assurée (Wilde, 2007). Seul un nombre très limité de centres de traitement antirabique propose ce produit à leurs patients (Dodet et al., 2008a, 2008b).

La majeure partie de la production d'HRIG est utilisée en Europe et en Amérique du Nord. Il existe dans ces pays un risque non négligeable de difficultés d'approvisionnement en cas d'augmentation rapide de la demande, par exemple lors de problèmes de rage de grande ampleur liée à des cas d'importation de chiens enragés en provenance de zones d'endozootie (Bourhy et al., 2009). Dans le reste du monde, les ERIG et les $\mathrm{F}(\mathrm{ab})_{2}$, dont le coût pour un traitement est environ dix fois moindre que celui des HRIG, sont utilisées.

Depuis la recommandation d'utiliser la dose de RIG au maximum en infiltration locale (Anonymous, 2005), aucune étude précise n'a essayé d'évaluer la quantité nécessaire. Les doses recommandées sont restées les mêmes que celles des protocoles utilisant une injection intramusculaire à distance, ce qui entraîne très probablement un surdosage et donc une surconsommation, ce qui est paradoxal en ce qui concerne un tel produit, précieux et coûteux (Anderson, 2007). De plus, un mauvais usage des immunoglobulines a été noté, y compris dans de nombreux pays industrialisés (Folb \& Cooke, 2007; Gautret et al., 2008).

L'avenir repose sur une meilleure rationalisation de l'usage des immunoglobulines antirabiques, en particulier dans les pays développés (Bourhy et al.,
2009), et sur l'utilisation plus large d'ERIG purifiées ou de nouveaux produits actuellement en cours de développement.

\section{Les produits en développement}

La nécessité de développer de nouveaux produits de substitution pour permettre l'administration de RIG à l'ensemble des patients répondant aux critères correspondants de l'OMS est aujourd'hui largement reconnue. Les anticorps monoclonaux (AcM) murins et humains dirigés contre la glycoprotéine d'enveloppe ont depuis longtemps montré leur activité neutralisante et protectrice dans des modèles expérimentaux (Dietzschold et al., 1987, 1990; Schumacher et al., 1989, 1992; Marissen et al., 2005). L'utilisation des AcM murins est moins avancée et pose potentiellement des problèmes anaphylactiques (Muhamuda et al., 2007). La combinaison d'AcM humains présentant les mêmes capacités neutralisantes que les HRIG semble une bonne alternative (Dorfman et al., 1994; Champion et al., 2000; Bakker et al., 2005, 2008; Sloan et al., 2007). Ces anticorps sont sélectionnés pour présenter des spécificités et des fonctionnalités complémentaires. En clair, ils doivent présenter une efficacité élevée mesurée in vitro et chez l'animal, un spectre très large d'activité permettant de couvrir l'éventail potentiel de variants antigéniques de glycoprotéine de Lyssavirus auxquels les patients risquent d'être exposés, ne pas cibler le même épitope (ne pas entrer en compétition) et enfin ne pas présenter de risque accru d'interférence comparativement aux RIG actuellement utilisées (Goudsmit et al., 2006). Un cocktail de deux AcM a ainsi été récemment choisi (de Kruif et al., 2007). Bien que prometteurs, ces produits devraient bénéficier d'une autorisation de mise sur le marché au mieux en 2012-2013.

Des approches plus systématiques utilisant le génie génétique (Prosniak et al., 2003), et plus particulièrement la technologie des librairies immunes exposées à la surface de phages, ont permis d'obtenir des fractions d'anticorps actives très proches des anticorps humains sans les inconvénients des anticorps xénogéniques (Zhao et al., 2008).

\section{Conclusion}

L'immunothérapie antirabique passive fait partie intégrante de la PPE, et est indispensable en cas d'exposition à un risque de catégorie 3 . Les produits actuels, en particulier les immunoglobulines d'origine humaine et les fragments $\mathrm{F}(\mathrm{ab})_{2}$ obtenus chez le cheval, fabriqués selon les recommandations de l'OMS et contrôlés internationalement, présentent une très 
bonne efficacité et un taux de réactions adverses minime. Associées au vaccin selon des protocoles validés par l'OMS, les RIG procurent une protection quasi totale. Cependant, près de 60 ans après la première recommandation concernant leur usage par le Comité d'experts OMS, l'accessibilité à ces produits reste très limitée pour les populations à risques, mettant en péril la vie de nombreuses victimes. Il est nécessaire d'informer et d'éduquer le personnel et les autorités de santé sur la nécessité d'utiliser l'immunothérapie passive pour le succès de la PPE, et sur l'absence de risque lié à cet usage. Dans l'avenir, il faut espérer une augmentation des quantités disponibles de produits purifiés d'origine équine ainsi que la mise sur le marché de nouveaux produits issus des AcM et du génie génétique dont le coût pourrait être moindre.

\section{References}

Anderson D.C., WHO guidelines dealing with immunoglobulin use impede rabies prevention. Asian Biomedicine, 2007, 1, 103-107.

Anderson G.R., Sgouris J.T., The preparation of rabies immune globulin and use in monkeys following virus challenge, Symposia Series in Immunobiological Standardization, T. International Symposium on Rabies (Ed.), 1965, Karger, Basel, p. 319.

Anonymous., WHO Expert Consultation on rabies. In World Health Organ Tech Rep Ser, WHO, 2005, Geneva, pp. 1-88.

Archer B.G., Dierks R.E., Effects of homologous or heterologous antiserum on neutralizing-antibody response to rabies vaccine. Bull World Health Organ, 1968, 39, 407-417.

Atanasiu P., Bahmanyar M., Baltazard M., Fox J.P., Habel K., Kaplan M.M., Kissling R.E., Komarov A., Koprowski H., Lépine P., Perez Gallardo F., Schaeffer M., Rabies neutralizing antibody response to different schedules of serum and vaccine inoculations in nonexposed persons. 1. Bull World Health Organ, 1956, 14, 593-611.

Atanasiu P., Bahmanyar M., Baltazard M., Fox J.P., Habel K., Kaplan M.M., Kissling R.E., Komarov A., Koprowski H., Lépine P., Perez Gallardo F., Schaeffer M., Rabies neutralizing antibody response to different schedules of serum and vaccine inoculations in nonexposed persons. 2. Bull World Health Organ, 1957, 17, 911-932.

Atanasiu P., Cannon D.A., Dean D.J., Fox J.P., Habel K., Kaplan M.M., Kissling R.E., Koprowski H., Lépine P., Perez Gallardo F., Rabies neutralizing antibody response to different schedules of serum and vaccine inoculations in non-exposed persons. 3. Bull World Health Organ, 1961, 25, 103-114.

Atanasiu P., Dean D.J., Habel K., Kaplan M.M., Koprowski H., Lépine P., Serie C., Rabies neutralizing antibody response to different schedules of serum and vaccine inoculations in non-exposed persons. 4. Bull World Health Organ, 1967, 36, 361-365.

Babes V., Lepp M., Recherches sur la vaccination antirabique. Ann Inst Pasteur (Paris), 1889, 3, 385-390.

Babes V., Cherchez T., Expérience sur l'atténuation du virus fixe. Ann Inst Pasteur (Paris), 1891, 10, 625-632.

Badrane H., Bahloul C., Perrin P., Tordo N., Evidence of two Lyssavirus phylogroups with distinct pathogenicity and immunogenicity. J Virol, 2001, 75, 3268-3276.

Bahmanyar M., Results of rabies post-exposure treatment with antirabies serum and the human diploid cell vaccine in Iran. Dev Biol Stand, 1978, 40, 163-165.

Bahmanyar M., Fayaz A., Nour-Salehi S., Mohammadi M., Koprowski H., Successful protection of humans exposed to rabies infection. Postexposure treatment with the new human diploid cell rabies vaccine and antirabies serum. J Am Med Assoc, 1976, 236, 27512754 .

Bahmanyar M., Fayaz A., Nour-Salehi S., Mohammadi M., Koprowski H., Successful protection of humans exposed to rabies infection. Postexposure treatment with the new human diploid cell rabies vaccine and antirabies serum. Wilderness Environ Med, 2000, 11, 42-46.

Bakker A.B., Marissen W.E., Kramer R.A., Rice A.B., Weldon W.C., Niezgoda M., Hanlon C.A., Thijsse S., Backus H.H., de Kruif J., Dietzschold B., Rupprecht C.E., Goudsmit J., Novel human monoclonal antibody combination effectively neutralizing natural rabies virus variants and individual in vitro escape mutants. J Virol, 2005, 79, 9062-9068.

Bakker A.B., Python C., Kissling C.J., Pandya P., Marissen W.E., Brink M. F., Lagerwerf F., Worst S., van Corven E., Kostense S., Hartmann K., Weverling G.J., Uytdehaag F., Herzog C., Briggs D.J., Rupprecht C.E., Grimaldi R., Goudsmit J., First administration to humans of a monoclonal antibody cocktail against rabies virus: safety, tolerability, and neutralizing activity. Vaccine, 2008, 26, 5922-5927.

Baltazard M., Bahmanyar M., Field trials with rabies vaccine on persons bitten by rabid wolves. Bull World Health Organ, 1955, 13, 747-772.

Benmansour A., Leblois H., Coulon P., Tuffereau C., Gaudin Y., Flamand A., Lafay F., Antigenicity of rabies virus glycoprotein. J Virol, 1991, 65, 4198-4203.

Bourhy H., Dacheux L., Strady C., Mailles A., Rabies in Europe in 2005. Euro Surveill, 2005, 10, 213-216.

Bourhy H., Goudal M., Mailles A., Sadkowska-Todys M., Dacheux L., Zeller H., Is there a need for anti-rabies vaccine and immunoglobulins rationing in Europe? Euro Surveill, 2009, 14.

Cabasso V.J., Properties of rabies immune globulin of human origin. J Biol Stand, 1974, 2, 43-50.

Cabasso V.J., Dobkin M.B., Roby R.E., Hammar A.H., Antibody response to a human diploid cell rabies vaccine. Appl Microbiol, 1974, 27, 553-561.

Cabasso V.J., Loofbourow J.C., Roby R.E., Anuskiewicz W., Rabies immune globulin of human origin: preparation and dosage determination in non-exposed volunteer subjects. Bull World Health Organ, 1971, 45, 303-315. 
Champion J.M., Kean R.B., Rupprecht C.E., Notkins A.L., Koprowski H., Dietzschold B., Hooper D.C., The development of monoclonal human rabies virusneutralizing antibodies as a substitute for pooled human immune globulin in the prophylactic treatment of rabies virus exposure. J Immunol Methods, 2000, 235, 81-90.

Coutard B., Gorbalenya A.E., Snijder E.J., Leontovich A.M., Poupon A., de Lamballerie X., Charrel R., Gould E.A., Gunther S., Norder H., Klempa B., Bourhy H., Rohayem J., L'Hermite E., Nordlund P., Stuart D.I., Owens R.J., Grimes J.M., Tucker P.A., Bolognesi M., Mattevi A., Coll M., Jones T.A., Aqvist J., Unge T., Hilgenfeld R., Bricogne G., Neyts J., La Colla P., Puerstinger G., Gonzalez J.P., Leroy E., Cambillau C., Romette J.L., Canard B., The VIZIER project: preparedness against pathogenic RNA viruses. Antiviral Res, 2008, 78, 37-46.

Cupo P., Azedevo-Marques M.M., Sarti W., Hering S.E., Proposal of abolition of the skin sensitivity test before equine rabies immune globulin application. Rev Inst Med Trop Sao Paulo, 2001, 43, 51-53.

Dacheux L., Reynes J.M., Buchy P., Sivuth O., Diop B.M., Rousset D., Rathat C., Jolly N., Dufourcq J.B., Nareth C., Diop S., Iehle C., Rajerison R., Sadorge C., Bourhy H., A reliable diagnosis of human rabies based on analysis of skin biopsy specimens. Clin Infect Dis, 2008, 47, 1410-1417.

de Kruif J., Bakker A.B., Marissen W.E., Kramer R.A., Throsby M., Rupprecht C.E., Goudsmit J., A human monoclonal antibody cocktail as a novel component of rabies postexposure prophylaxis. Annu Rev Med, 2007, 58, 359-568.

Dean D., Local wound treatment of animal bites, Proc. Nat. Rabies Symposium, Nat. Commun. Dis. Center, 1966, Atlanta, Georgia, p. 85.

Dean D.J., Baer G.M., Thompson W.R., Studies on the local treatment of rabies-infected wounds. Bull World Health Organ, 1963, 28, 477-486.

Delmas O., Holmes E.C., Talbi C., Larrous F., Dacheux L., Bouchier C., Bourhy H., Genomic diversity and evolution of the lyssaviruses. PLoS ONE, 2008, 3, e2057.

Dietzschold B., Gore M., Casali P., Ueki Y., Rupprecht C.E., Notkins A.L., Koprowski H., Biological characterization of human monoclonal antibodies to rabies virus. J Virol, 1990, 64, 3087-3090.

Dietzschold B., Tollis M., Lafon M., Wunner W.H., Koprowski H., Mechanisms of rabies virus neutralization by glycoprotein-specific monoclonal antibodies. Virology, 1987, 161, 29-36.

Dodet B., Adjogoua E.V., Aguemon A.R., Amadou O.H., Atipo A.L., Baba B.A., Ada S.B., Boumandouki P., Bourhy H., Diallo M.K., Diarra L., Diop B. M., Diop S.A., Fesriry B., Gosseye S., Hassar M., Kinge T., Kombila Nzamba T.E., Yandoko E.N., Nzengue E., Ramahefalalao E.F., 2nd, Ratsitorahina M., Simpore L., Soufi A., Tejiokem M., Thiombano R., Tiembre I., Traore A.K., Wateba M.I., Fighting rabies in Africa: the Africa Rabies Expert Bureau (AfroREB). Vaccine, 2008a, 26, 6295-6298.
Dodet B., Goswami A., Gunasekera A., de Guzman F., Jamali S., Montalban C., Purba W., Quiambao B., Salahuddin N., Sampath G., Tang Q., Tantawichien T., Wimalaratne O., Ziauddin A., Rabies awareness in eight Asian countries. Vaccine, 2008b, 26, 6344-6348.

Dorfman N., Dietzschold B., Kajiyama W., Fu Z.F., Koprowski H., Notkins A.L., Development of human monoclonal antibodies to rabies. Hybridoma, 1994, 13, 397-402.

Fernandes A., Kaundinya J.O., Daftary G., Saxena L., Banerjee S., Pattnaik P., Chromatographic purification of equine immunoglobulin $\mathrm{G} \mathrm{F}(\mathrm{ab})_{2}$ from plasma. J Chromatogr B Analyt Technol Biomed Life Sci, 2008, 876, 109-115.

Folb J.E., Cooke R.P., Issues of human rabies immunoglobulin and vaccine: policy versus practice. J Public Health (Oxf), 2007, 29, 83-87.

Gautret P., Soula G., Adamou H., Soavi M.J., Delmont J., Rotivel Y., Parola P., Brouqui P., Rabies postexposure prophylaxis, Marseille, France, 1994-2005. Emerg Infect Dis, 2008, 14, 1452-1454.

Glück R., Labert D., Techniques de purification de l'immunsérum antirabique hétérologue. La rage: techniques de laboratoire, Meslin F.-X, Kaplan M.M.,. Koprowski H (Eds.), 1999, Organisation Mondiale de la Santé, Genève, pp. 411-416.

Goudsmit J., Marissen W.E., Weldon W.C., Niezgoda M., Hanlon C.A., Rice A.B., Kruif J., Dietzschold B., Bakker A.B., Rupprecht C.E., Comparison of an anti-rabies human monoclonal antibody combination with human polyclonal anti-rabies immune globulin. $J$ Infect Dis, 2006, 193, 796-801.

Habel K., Seroprophylaxis in experimental rabies. Pub Hlth Rep, 1945, 60, 545.

Hanlon C.A., Kuzmin I.V., Blanton J.D., Weldon W.C., Manangan J.S., Rupprecht C.E., Efficacy of rabies biologics against new lyssaviruses from Eurasia. Virus Res, 2005, 111, 44-54.

Hattwick M.A.W., Rubin R.H., Music S., Sikes R.K., Smith J.S., Gregg M. D., Postexposure rabies prophylaxis with human rabies immune globulin. J Am Med Assoc, 1974, 227, 407.

Helmick C.G., Johnstone C., Sumner J., Winkler W.G., Fager S., A clinical study of Merieux human rabies immune globulin. J Biol Stand, 1982, 10, 357-367.

Hosty T.S., Kissling R.E., Schaeffer M., Wallace G.A., Dibble E.H., Human antirabies gamma globulin. Bull World Health Organ, 1959, 20, 1111-1119.

Kaplan M.M., Cohen D., Koprowski H., Dean D., Ferrigan, L., Studies on the local treatment of wounds for the prevention of rabies. Bull World Health Organ, 1962, 26, 765-775.

Kaplan M.M., Paccaud M.F., Effectiveness of locally inoculated antirabies serum and gamma-globulin in rabies infection of mice. Bull World Health Organ, 1963, 28, 495-497.

Karliner J.S., Belaval G.S., Incidence of reactions following administration of antirabies serum; study of 526 cases. J Amer Med Assoc, 1965, 193, 359-362. 
Knobel D.L., Cleaveland S., Coleman P.G., Fevre E.M., Meltzer M.I., Miranda M.E., Shaw A., Zinsstag J., Meslin F.X., Re-evaluating the burden of rabies in Africa and Asia. Bull World Health Organ, 2005, 83, 360-368.

Koprowski H., Van der Scheer J., Black J., Use of hyperimmune antirabies serum concentrates in experimental animals. Am J Med, 1950, 8, 412.

Kuzmin I.V., Wu X., Tordo N., Rupprecht C.E., Complete genomes of Aravan, Khujand, Irkut and West Caucasian bat viruses, with special attention to the polymerase gene and non-coding regions. Virus Res, 2008,136, 81-90.

Lafon M., Edelman L., Bouvet J.P., Lafage M., Montchatre E., Human monoclonal antibodies specific for the rabies virus glycoprotein and N protein. J Gen Virol, 1990, 71, 1689-1696.

Lang J., Attanath P., Quiambao B., Singhasivanon V., Chanthavanich P., Montalban C., Lutsch C., PepinCovatta S., Le Mener V., Miranda M., Sabchareon A., Evaluation of the safety, immunogenicity, and pharmacokinetic profile of a new, highly purified, heattreated equine rabies immunoglobulin, administered either alone or in association with a purified, Vero-cell rabies vaccine. Acta Trop, 1998, 70, 313-333.

Luekrajang T., Wangsai J., Phanuphak P., Production de sérum antirabique d'origine équine, La rage: techniques de laboratoire, Meslin F.-X., Kaplan M.M., Koprowski H. (Eds.), 1999, Organisation Mondiale de la Santé, Genève, pp. 407-410.

Marie A., Recherches sur le sérum antirabique. Ann Inst Pasteur (Paris), 1908, 22, 271.

Marissen W.E., Kramer R.A., Rice A., Weldon W.C., Niezgoda M., Faber M., Slootstra J.W., Meloen R.H., Clijsters-van der Horst M., Visser T.J., Jongeneelen M., Thijsse S., Throsby M., de Kruif J., Rupprecht C.E., Dietzschold B., Goudsmit J., Bakker A.B., Novel rabies virus-neutralizing epitope recognized by human monoclonal antibody: fine mapping and escape mutant analysis. J Virol, 2005, 79, 4672-4678.

Muhamuda K., Madhusudana S.N., Ravi V., Use of neutralizing murine monoclonal antibodies to rabies glycoprotein in passive immunotherapy against rabies. Hum Vaccin, 2007, 3, 192-195.

OMS, Comité d'expert de la rage. G. (Ed.), Série de rapports techniques. Deuxième rapport, Organisation Mondiale de la Santé, Genève, 1954.

Proca G., Bobes S., Immunisation contre la rage: vaccins vivants et vaccins tués. Bull Org Hyg SDN, 1940, 9, 85-137.

Prosniak M., Faber M., Hanlon C.A., Rupprecht C.E., Hooper D.C., Dietzschold B. Development of a cocktail of recombinant-expressed human rabies virusneutralizing monoclonal antibodies for postexposure prophylaxis of rabies. J Infect Dis, 2003,188, 53-56.

Quiambao B.P., Dytioco H.Z., Dizon R.M., Crisostomo M.E., Laot T.M., Teuwen D.E., Rabies post-exposure prophylaxis in the Philippines: health status of patients having received purified equine $\mathrm{F}(\mathrm{ab}$ ') (2) fragment rabies immunoglobulin (Favirab). PLoS Negl Trop Dis, 2008, 2, e243.
Saesow N., Chaiwatanarat T., Mitmoonpitak C., Wilde H., Diffusion and fate of intramuscularly injected human rabies immune globulin. Acta Trop, 2000, 76, 289-292.

Schumacher C.L., Dietzschold B., Ertl H.C., Niu H.S., Rupprecht C.E., Koprowski H., Use of mouse antirabies monoclonal antibodies in postexposure treatment of rabies. J Clin Invest, 1989, 84, 971-975.

Schumacher C.L., Ertl H.C., Koprowski H., Dietzschold B., Inhibition of immune responses against rabies virus by monoclonal antibodies directed against rabies virus antigens. Vaccine, 1992, 10, 754-760.

Selimov M., Boltucij L., Semenova E., Kobrinskij G., Zmusko L., Results of the use of antirabies gamma glubulin and vaccine in prophylaxis of rabies in the Soviet Union. J Hyg Epidem Microbiol Immunol (Praha), 1959, 3, 168.

Sikes R.K., Human rabies immune globulin. Public Health Rep, 1969, 84, 797-801.

Sloan S.E., Hanlon C., Weldon W., Niezgoda M., Blanton J., Self J., Rowley K.J., Mandell R.B., Babcock G.J., Thomas W.D. Jr., Rupprecht C.E., Ambrosino D.M., Identification and characterization of a human monoclonal antibody that potently neutralizes a broad panel of rabies virus isolates. Vaccine, 2007, 25, 2800-2810.

Suwansrinon, K., Jaijareonsup W., Wilde H., Benjavongkulchai M., Sriaroon C., Sitprija V., Sex- and age-related differences in rabies immunoglobulin hypersensitivity. Trans $R$ Soc Trop Med Hyg, 2007, 101, 206-208.

Suwansrinon K., Jaijaroensup W., Daviratanasilpa S., Sriaroon C., Wilde H., Sitprija V. Adverse reactions to human rabies immune globulin manufactured by the Thai Red Cross Society. Vaccine, 2005, 23, 1324-1325.

WHO, Comité d'experts de la rage, G. (Ed.), Série de rapports techniques. Premier rapport, Organisation Mondiale de la Santé, Genève, 1950.

WHO, Who Expert Committee on Rabies, G. (Ed.) Who Technical Report Series. Sixth Report, World Health Organization, Genève, 1973.

WHO, Rabies vaccine. Position paper. Wkly Epidemiol Rec, 2007, 425-435.

Wiktor T.J., Lerner R.A., Koprowski H., Inhibitory effect of passive antibody on active immunity induced against rabies by vaccination. Bull World Health Organ, 1971, 45, 747-753.

Wilde H., Failures of post-exposure rabies prophylaxis. Vaccine, 2007, 25, 7605-7609.

Willoughby R.E.Jr., Tieves K.S., Hoffman G.M., Ghanayem N.S., Amlie-Lefond C.M., Schwabe M.J., Chusid M.J., Rupprecht C.E., Survival after treatment of rabies with induction of coma. $N$ Engl J Med, 2005, 352, 2508-2514.

Winkler W.G., Schmidt R.C., Sikes R.K. Evaluation of human rabies immune globulin and homologous and heterologous antibody. J Immunol, 1969, 102, 1314-1321.

Zhao X.L., Yin J., Chen W.Q., Jiang M., Yang G., Yang Z.H., Generation and characterization of human monoclonal antibodies to G5, a linear neutralization epitope on glycoprotein of rabies virus, by phage display technology. Microbiol Immunol, 2008, 52, 89-93. 